\title{
El papel de la Ciencia en el proceso de Ordenamiento Territorial (y en otras cuestiones vinculadas con problemas ambientales)
}

\author{
José M. PARUelo ${ }^{1 / 2}$ \\ ${ }^{1}$ Laboratorio de Análisis Regional y Teledetección, Depto. Métodos Cuantitativos y Sistemas de Información. IFEVA y \\ Facultad de Agronomía. CONICET y UBA. Ciudad Autónoma de Buenos Aires, Argentina. ${ }^{2}$ Instituto de Ecología y Ciencias \\ Ambientales. Facultad de Ciencias. Universidad de la República. Montevideo, Uruguay.
}

\begin{abstract}
Resumen. Los aspectos técnicos, políticos y administrativos del Ordenamiento Territorial Rural (OTR) están estrechamente enlazados, y difícilmente se pueden tratar de forma aislada. Incorporar a la Ciencia y a los científicos en el proceso de OTR permite mejorar algunos factores que definen la calidad del proceso (e.g., acceso a la información, percepción de los problemas, etc.), pero no otros (e.g., disputas de valores e intereses o asimetrías de poder). En su condición de conocimiento sistematizado, la Ciencia aporta un "banco de conocimientos" indispensable. A su vez, puede jugar otro papel clave al ofrecer esquemas de pensamiento y acción, en los cuales los criterios de verdad se asocian a evidencias empíricas y lógicas y no al principio de autoridad o al pensamiento mágico. La sociedad y el Sistema de Ciencia y Tecnología (SCyT) deberían identificar los casos en los que la participación es crítica y se requiere superar debates poco informados. La participación de la Ciencia y los científicos es clave cuando se debe disminuir la incertidumbre asociada a la toma de decisiones. Por su parte, se debería evitar la situación, menos reconocida, de forzar la incorporación del SCyT cuando no corresponde. La participación de los científicos tiene que ocurrir en colaboración estrecha con otros actores del proceso. No obstante, su participación en los procesos de OTR enfrenta muchos desafíos y problemas. Una lista no exhaustiva incluye los conflictos de interés, el reduccionismo y la consecución de la inter / trans / multidisplinariedad.
\end{abstract}

[Palabras clave: planificación, Sistema de Ciencia y técnica, política, toma de decisiones]

\begin{abstract}
AвSTRACT. The role of Science in land use planning (and in some other issues related to environmental problems). The technical, political and administrative aspects of Rural Land Planning are closely linked and hardly can be treated in isolation. To incorporate science and scientists in land planning improves some factors that define the quality of the process (e.g. access to information, perception of problems, etc.) but not others (disputes on values and interests or power asymmetries). Science incorporates, as systematized knowledge, an indispensable "knowledge bank". In turn, Science can play another key role in providing patterns of thought and action in which the criteria of truth are associated with empirical and logical evidence and not to the principle of authority or magical thinking. Society and the System of Science and Technology (SCyT) should identify those cases where participation is critical to avoid uninformed debates. The involvement of Science and scientists is important when you have to reduce the uncertainty associated with decision making. Should be avoided, meanwhile, the least recognized situation to force the participation of the SCyT in the cases of disputes over interests and values. In such situations its participation becomes unnecessary and possibly counterproductive. The participation of scientists has to happen in close collaboration with other stakeholders. The participation of the SCyT in the land planning processes faces, however, many challenges and problems. A non-exhaustive list includes conflicts of interest, reductionism and the possibility to achieve inter / trans / multidisplinarity.
\end{abstract}

[Keywords: planning, Science and technology systems, policies, politics, decision making]

\section{INTRODUCCIÓN}

El ordenamiento del territorio, y en particular el rural, es un tema que ha ganado importancia en la discusión pública en la Argentina y en países vecinos (Paruelo et al. 2015; GarcíaCollazo etal. 2013). Las definiciones del proceso comúnmente denominado Ordenamiento Territorial Rural (OTR) (Paruelo et al. 2015) enfatizan la existencia de un componente técnico, uno político y uno administrativo. La importancia relativa de cada uno de esos

Editor asociado: Esteban Jobbágy

$\triangle$ paruelo@agro.uba.ar componentes varía en las distintas etapas del proceso. En la etapa de caracterización del sistema territorial o socioecológico, el componente técnico tiene un peso mayor que el administrativo y el político. En la definición de metas y objetivos, la dimensión política es la más relevante, mientas que los administrativos dominan la etapa de implementación de los planes. Más allá de estos cambios en el peso relativo los tres componentes, todos están operando a lo largo del proceso. Más aun, los aspectos técnicos, políticos y administrativos

Recibido: 22 de octubre de 2015

Aceptado: 14 de diciembre de 2015 
están muy enlazados y, difícilmente pueden aislarse entre sí. Así, la caracterización de los recursos edáficos, un tema eminentemente técnico, dependerá de los usos actuales y futuros del suelo. Por ejemplo, la perspectiva de hacer o no cultivos de riego determinará en qué aspectos de la caracterización de las propiedades físicas del suelo se pondrá énfasis. Los valores e intereses de determinados grupos o actores sociales determinarán qué poblaciones animales y vegetales relevar de manera prioritaria. Los modelos de desarrollo económico que los distintos actores tengan en mente condicionarán la percepción de procesos de degradación del suelo o la vegetación. El reemplazo o la introducción de especies forrajeras en un pastizal natural se podrán percibir como una mejora o como degradación de un ecosistema natural. Al igual que en el caso de la salud pública, los aspectos técnicos o científicos en un proceso de OTR están muy influenciados por aspectos políticos (e.g., relaciones de poder e intereses sectoriales) y/o ideológicos (el papel del Estado en la economía o los valores a promover con una ley). En el caso de la salud pública, la certeza acerca de las consecuencias del consumo de tabaco sobre la salud humana no evitó que durante muchos años los intereses económicos y el peso político de las tabacaleras impidieran la generalización de campañas anti-tabaco, el endurecimiento de las restricciones a los lugares en donde fumar o la prohibición de la publicidad.

Una serie de factores complican un abordaje "aséptico" desde el Sistema de Ciencia y Técnica (SCyT) para lograr un OTR inteligente, justo y útil. Estos incluyen: i) las disputas de valores e intereses, ii) las asimetrías de poder, iii) las asimetrías en el acceso a la información, iv) los problemas asociados a la percepción cambiante según la escala temporal y espacial en la que se desenvuelven los actores e involucrados, y v) las diferencias en las cosmovisiones entre actores sociales y políticos. El SCyT incluye las instituciones públicas y privadas dedicadas a la generación y la transferencia de conocimiento científico. Al referirnos a un sistema incluimos los recursos físicos, institucionales, relaciones y por supuesto al personal. Al incorporar al personal, esto es los investigadores y técnicos, incluimos además la diversidad de opiniones, valores e intereses que conviven dentro de la comunidad científica (Paruelo 2009).

Incorporar a la Ciencia y a los científicos en el proceso de OTR permite trabajar sobre algunos de los factores mencionados (acceso a la información, percepción de los problemas) pero no sobre otros (disputas de valores e intereses o asimetrías de poder). En este artículo analizamos cómo algunos fracasos pasados justifican o demandan la participación del SCyT en procesos de OTR. Por otra parte, diferenciamos el papel que los científicos y la Ciencia pueden jugar en el procesos de OTR, las dificultades de la relación entre el SCyT con otros actores y los posibles roles que los científicos pueden adoptar en un proceso con un fuerte componente político. Finalmente, discutimos algunos desafíos y propuestas.

\section{LAS EXPERIENCIAS HistÓRICAS DEL OTR POR PRESENCIA O AUSENCIA}

A lo largo de la historia de la humanidad, los procesos de OTR han tenido lugar de manera más o menos espontánea siguiendo distintos modelos. En algunos casos, el resultado del OTR fue duradero (miles de años). Las chinampas en México (Ezcurra 1998) o la agricultura intensiva en las tierras altas de Nueva Guinea (Diamond 2006) representan sistemas basados sobre una cuidadosa planificación del territorio y han mostrado su capacidad de perdurar. Muchos "consorcios de riego" desarrollados por los pueblos árabes en la cuenca del Mediterráneo hace siglos siguen funcionando en el presente. Estos ejemplos fueron, en muchos casos, generados a partir de acuerdos de habitantes a partir de una aproximación de abajo hacia arriba ("bottom-up"), propiciando sistemas de gobernanza muy estables (Ostrom 1999). En general, y debido al escaso desarrollo del SCyT en las sociedades involucradas en los ejemplos citados, la aproximación para llegar a un diseño de uso del territorio fue la prueba y el error.

Por otro lado, muchas sociedades desarrollaron formas de usar el territorio que generaron problemas ambientales muy serios y que, junto a otros factores, determinaron el colapso de sus sistemas socioecológicos (Redman 1999). Por pérdida de capital natural, social, humano o cultural estos sistemas no fueron capaces de adaptarse y/o no manifestaron un comportamiento resiliente que les permitiera mantenerse. Redman (1999) y Diamond (2006) hacen un análisis pormenorizado de una serie de sistemas socioecológicos que colapsaron en distintos contextos geográficos e históricos, 
desde la población maorí de la Isla Pascua hasta la colonización noruega de Groenlandia, pasando por la cultura Anasazi en el desierto Chihuaense de Norte América a los Mayas en la península de Yucatán. De esos casos estos autores identifican una serie de factores que contribuyeron a desencadenar el colapso.

1. El modelo usado para tomar decisiones se basa en el conocimiento ganado en otros ecosistemas o en otro momento con similitudes superficiales, pero diferencias críticas. Por ejemplo, el modelo de cría de ganado ovino en la Patagonia instalado a principios del siglo XX se basó en la importación de un modelo exitoso en las islas británicas y terminó generando un serio proceso de desertificación (Paruelo \& Aguiar 2003).

2. Se cuenta con información insuficiente y se sobregeneraliza el problema y/o se cuenta con una descripción pobre de la heterogeneidad espacial. La difusión de pasturas implantadas con especies exóticas en la década del setenta en la Pampa Inundable sin reconocer la heterogeneidad de ambientes llevó a que se degraden pastizales naturales, reemplazados por pasturas menos productivas.

3. La definición del problema es deficiente. Por ejemplo, debido a que no se identifican las verdaderas causas de las situaciones problemáticas, como ocurre con frecuencia cuando se adjudican a factores puramente climáticos las inundaciones que son propiciadas también por cambio en el uso del suelo (Nosetto et al. 2015).

4. El período de observación del sistema es corto y no se captan aspectos fundamentales de la dinámica $o$ tendencias. La difusión de la agricultura de secano en zonas semiáridas durante períodos húmedos y su colapso en ciclos secos es un ejemplo. El aumento lento pero sostenido del nivel de napas freáticas en áreas en donde se reemplazan bosques por cultivos y los procesos de salinización que de ellos derivan, son un ejemplo (Santoni et al. 2010; Amdan et al. 2013).

5. Quienes manejan y toman decisiones no son quienes efectivamente gestionan y perciben los recursos. El proceso de "land grabbing" (adquisición y/o manejo de tierras agrícolas y forestales en países de América latina, África y el sudeste de Asia por parte de corporaciones multinacionales) ubica a los tomadores de decisión no ya fuera del territorio sino en otro continente (Rulli et al. 2013).
6. La reacción de quienes toman decisiones está desfasada de la dinámica del proceso de deterioro y se termina haciendo "demasiado poco y demasiado tarde". Un aspecto muy relacionado con el punto anterior.

7- Los que manejan y toman decisiones pueden percibir el problema pero les resulta ajeno. La pérdida de biodiversidad o la emisión de gases con efecto invernadero pueden ser percibidas por los tomadores de decisión como un problema de los gobiernos o, más aún, de organismos internacionales.

8. El "modelo" de naturaleza del cual se parte es deficiente. Un caso típico es asumir una dinámica de equilibrio en la planificación y la evaluación de impactos. Una serie de factores exógenos (desde los cambios climáticos a las invasiones biológicas) o endógenos (la dinámica sucesional de un bosque, el régimen de perturbaciones) hacen que el cambio sea la constante.

Los ejemplos que acompañan la lista muestran que estos factores siguen operando en sistemas que nos resultan familiares. Los factores 1, 2, 3, 4 y 8 se relacionan con falta de información, con una mala sistematización y análisis de esa información o con modelos conceptuales deficientes del sistema socioecológico. Para todos estos factores, la Ciencia, en tanto conocimiento estructurado y sistematizado, puede convertirse en un auxiliar clave del proceso de OTR. En el resto de los problemas, de naturaleza política y vinculada a la gobernanza, el papel del SCyT es importante pero no directo.

\section{La Ciencia y los Científicos en el OTR}

Separemos por un lado el aporte de la Ciencia al proceso de OTR y por el otro el de los científicos. La Ciencia aporta, en su condición de conocimiento sistematizado, un "banco de conocimientos" indispensable. Esto incluye información, pero también modelos conceptuales. No accedemos a ese banco sólo para "extraer" datos (e.g., la tasa de crecimiento esperable para un bosque determinado o el posible rendimiento de un cultivo), sino también modelos para interpretar la realidad. La relación diversidad-área de un parche de hábitat que postularon McArthur \& Wilson (1972) en su Teoría de Biogeografía de Islas sigue siendo un modelo indispensable para 
interpretar qué pasará con la riqueza de aves o mamíferos si se fragmenta un paisaje. Acceder a este "banco de conocimientos" requiere, obviamente, un entrenamiento riguroso.

Detrás de la idea del "banco de conocimiento" está el modelo "linear" de conexión del SCyT con la sociedad (Pielke Jr. 2007). De esta manera, la "ciencia básica" deposita en el banco de conocimientos, mientras que la "ciencia aplicada" genera desarrollos que se transforman en productos o procesos que, en última instancia, generan beneficios para la sociedad. Este modelo ha sido exitoso en muchas circunstancias (y seguramente lo seguirá siendo en otras). Sin embargo, en su forma idealizada produce una suerte de disociación entre la generación del conocimiento y las necesidades de la sociedad. El modelo es de una sola vía y el conocimiento fluye "aguas abajo", hacia la sociedad. Volveremos sobre esto.

La Ciencia puede jugar otro papel clave como una forma de pensar y actuar (Cereijido 1994). Frente a situaciones problemáticas, un enfoque científico permite generar hipótesis acerca de las causas de esas situaciones y sobre sus eventuales consecuencias. Este mecanismo permite pasar a la formulación de los "problemas" más relevantes a enfrentar. De estas hipótesis se pueden derivar predicciones contrastables con evidencias empíricas que ayudan a discriminar entre explicaciones alternativas. Un enfoque científico se define por usar las evidencias empíricas como criterio de verdad. Esto resulta clave en procesos de OTR en los que se suelen invocar las tradiciones o el principio de autoridad (lo dice un determinado caudillo político, líder religioso, un propietario importante o incluso un académico renombrado) para establecer la veracidad de algunas afirmaciones (Cereijido \& Reinking 2005).

Obviamente, los científicos, o sea aquellos que se dedican de manera profesional a la investigación en el marco del SCyT, se constituyen en intermediarios privilegiados entre el "banco de conocimientos" y la sociedad. Más aun, pueden hacer contribuciones específicas al "banco de conocimiento" en relación con un sistema territorial en particular (e.g., relevando la vegetación y los suelos del área a ser ordenada). El entrenamiento y la familiaridad con algunos temas permiten a los científicos, por ejemplo, extrapolar experiencias de otros ámbitos, adaptar modelos conceptuales y promover aproximaciones novedosas para la caracterización del sistema socioecológico, la evaluación de impactos o el monitoreo de los recursos (y generar así alternativas de uso del territorio novedosas).

Cereijido (1994) hace una distinción interesante entre científicos e investigadores, apuntando a que no todos quienes se dedican a generar conocimiento de manera experimental tienen una enfoque científico (serían sólo investigadores) y continúan basándose en prejuicios o el principio de autoridad en lugar de formular hipótesis, predecir y evaluar sobre la base de evidencia. Por otra parte, señala que un enfoque científico para percibir la realidad no es, de ninguna manera, una prerrogativa de los académicos.

La participación del SCyT no puede estar aislada de los otros actores. Esto determina que el modelo lineal muestre limitaciones en un proceso de OTR. Como señalábamos antes, los componentes técnicos, políticos y administrativos del OTR están unidos de manera intrincada (Latour 2012). El modelo lineal empieza a desdibujarse en la medida en que las necesidades, problemas, valores e intereses entran en juego. Entonces aparecen retrocontroles desde las necesidades y problemas a la investigación "básica". Por ello, la participación de los científicos tiene que ocurrir en estrecha colaboración con otros actores del proceso. Incluso, es crítico que la sociedad reconozca la importancia de incorporar al SCyT y que éste no se incorpore como una imposición.

La participación de la Ciencia y los científicos es importante cuando hay que disminuir la incertidumbre asociada a la toma de decisiones. Estas situaciones incluyen la falta de información, de modelos conceptuales y de alternativas de uso. Por lo general, se asocia a situaciones que, además, no involucran disputas de valores entre actores y las dudas acerca de la relación entre las acciones propuestas y los resultados son bajas (Pielke Jr. 2007).

Por otro lado, hay situaciones en la que se fuerza una solución provista por el SCyT cuando no corresponde. Este es el caso en el que se disputan intereses y/o valores (por ejemplo, es más importante preservar una especie o promover un desarrollo inmobiliario). Cuando 
las decisiones tienen que ver con disputas en torno a intereses y valores la participación del SCyT debería restringirse a identificar falacias o justificaciones técnicas que confunden o enmascaran motivaciones políticas.

\section{LA RELACión ENTRE EL SCyT y LOS Otros Actores del Proceso}

La relación del SCyT con el resto de los actores que participan con un proceso de OTR no es sencilla ni está exenta de conflictos. En el centro de esa desconexión están las diferencias en normas culturales y estilos de comunicación del SCyT y, en particular, de los sectores político-administrativos. El SCyT tiene especial dificultad para manejar situaciones de compromiso que involucran, además de evidencias objetivas, pautas culturales, tradiciones, intereses ocultos o pobremente formulados. Los técnicos o académicos involucrados en la formulación de una alternativa o de un diagnóstico a menudo suelen retirar su apoyo o perder motivación por participar cuando sus propuestas no son consideradas o resultan descartadas por otros actores. Latour (2012) advierte sobre estos conflictos y resalta la importancia que tienen no sólo la evidencia empírica o lógica para instalar un argumento en el seno de la sociedad, sino también el de la retórica (o sea, el de los procedimientos y técnicas de utilización del lenguaje, puestos al servicio de una finalidad persuasiva). En un proceso colectivo de convencimiento, las evidencias deben ser trabajadas para que los enunciados seas aceptados como verdaderos por el conjunto. Las evidencias se tornan "evidentes" luego de un proceso de argumentación y persuasión. Muchas veces, el sector académico no está preparado para esto y desprecia o toma con desdén esta labor.

Engeneral,las formulaciones quehaceelSCyT se expresan en términos de probabilidades ya que se asocian a situaciones de incertidumbre (i.e., aquellas en las que más de un resultado es consistente con nuestras expectativas [la acción de proteger un área puede o no evitar la extinción de la especie A]). Las incertidumbres (o riesgos, cuando hablamos del resultado valuado como negativo) asociadas a determinadas acciones sobre el territorio se expresan como probabilidades de extinción de una especie, pérdida de cosecha, recurrencia de inundaciones, entre otros ejemplos. En
Ciencia se asume que ciertos tipos de eventos son aleatorios y que no es posible prever el resultado de una acción. Dicho de otro modo, no todos los eventos son determinísticos. Las causas que subyacen al comportamiento aleatorio pueden ser variadas (Mahner \& Bunge 1997), y entre ellas se encuentran la complejidad (muchos componentes y relaciones no-lineales en los sistemas) y la falta de información. La existencia de aleatoriedad no implica que el mundo sea caótico o absolutamente indeterminado. Una serie de factores condicionan la ocurrencia de otros, y es posible describir grados en términos de probabilidad de ocurrencia de un evento. El concepto de probabilidad, en realidad, hace referencia a una serie de nociones como la incertidumbre, plausibilidad y credibilidad. De hecho, el valor de probabilidad que se asigna al resultado de un evento puede tener distintos orígenes. En un caso se asocia probabilidad a la frecuencia esperada de un evento (como en la cara o ceca al lanzar una moneda al aire). También es la manera en la cual se define la probabilidad de extinción de una especie en un dado contexto (haciendo muchas simulaciones de la dinámica poblacional, en las cuales una serie de factores varían de forma aleatoria y otros están determinados). En otros casos, la probabilidad surge de una evaluación subjetiva de las posibilidades de ocurrencia de un evento. En esta evaluación se integra la información y evidencias disponibles, asignándole una ponderación que resulta de los marcos conceptuales e ideológicos de quien lo realiza. Este último mecanismo de asignación de probabilidades a un evento es el que se conoce como "opinión de expertos".

Los sectores político-administrativos tienen una dificultad marcada para operar con la incertidumbre y el largo plazo. En muchos casos, el manejo del concepto de probabilidad de ocurrencia de un evento por parte de los actores es deficiente. La expectativa de "certeza científica" de distintos actores se ve frecuentemente frustrada. Detrás de esto hay razones fundamentales (e.g., el manejo deficiente de los conceptos de probabilidad, pronóstico, escenario y predicción) pero también otras asociadas a la comunicación y a la retórica. Se puede enfatizar la incertidumbre asociada a cuánto aumentará la temperatura en los próximos 30 años o la certidumbre de que aumentará. Sin duda la incorporación de información con probabilidades asociadas a la planificación es un tema que necesita ser 
trabajado con especial cuidado en el proceso de OTR.

El dinamismo de los sistemas complejos está claramente incorporado a la visión que se tiene en el ámbito científico. Esto se asocia a modelos de la naturaleza de tipo resilientes o evolutivos (Gunderson \& Holling 2002). Muchos actores perciben a los sistemas socioecológicos como "planos" y, por consiguiente, asumen que pueden "llevarlos" al estado que deseen a través de intervenciones mediadas por la tecnología. Otros actores, por ejemplo grupos ambientalistas, tienen una visión caótica de la naturaleza, en la cual cualquier intervención puede llevar al sistema al colapso. Modelos de naturaleza distintos y no explícitos pueden resultar en un serio problema de comunicación.

La participación del SCyT en proceso de OTR y en formas simples o parciales de éste (e.g., en evaluaciones de impacto ambiental o zonificaciones) estuvo formalmente vedada mediante mecanismos formales, muchas veces impuestos desde organismos internacionales como el BID o el Banco Mundial. Una visión conspirativa vería una exclusión activa del SCyT de procesos de toma de decisión. La participación de las universidades o institutos de investigaciones estuvo restringida (o directamente impedida) privilegiándose a firmas comerciales (agencias consultoras privadas). Si bien en muchas ocasiones las "consultoras" terminan contratando a miembros del SCyT, las posibilidades de influir y aportar a la toma de decisiones se ven seriamente restringida al quedar mediadas por estas firmas. La monopolización del trabajo técnico asociado al diagnóstico y planificación en cuestiones ambientales por parte de consultoras es un serio problema para garantizar la calidad de los productos, la transparencia de los procesos y la posibilidad de fortalecer a actores sociales postergados.

\section{Roles de Participación de los Académicos en el Proceso de OTR}

En su libro "The Honest Broker: Making sense of science in policy and politics" Roger Pielke Jr. (2007) propone cuatro roles para los científicos en su relación con la sociedad. El modelo lineal, que plantea un ámbito para la "ciencia básica" y otro para la "aplicada", define "a priori" un rol para los académicos: el del "científico puro" o el "árbitro científico".
En el primer caso, el científico se pone al margen de la disputa de valores e intereses. $\mathrm{Al}$ publicar sus artículos realiza su (valiosa) contribución al "banco de conocimientos". Su relación con los actores del proceso de OTR es nula y no tiene responsabilidad sobre cómo se usa el conocimiento generado.

El rol de "arbitro científico" también evita la interacción con los actores, pero admite la discusión con los tomadores de decisión acerca de cuestiones particulares o formar parte de paneles de evaluación. Básicamente, está dispuesto a discutir si "la opción A es mejor que la B para relevar la vegetación de este área" o "cuáles son los antecedentes respecto al efecto de las técnicas existentes de control de plagas sobre la salud de la fauna nativa", pero no a participar de la discusión que lleve a la toma de decisión con todos los involucrados.

Los miembros del SCyT pueden adoptar roles que implican una interacción fuerte con los actores del proceso y una participación activa en la disputa por valores e intereses. Esto plantea modelos más complejos de relación entre los científicos y los actores sociales y económicos que los propuestos por el esquema "lineal". El científico puede constituirse en un "abogado" de causas específicas. Por ejemplo, cuando un grupo académico a partir de los riesgos que una práctica representa para la salud humana o la supervivencia de una especie promueve activamente la prohibición de dicha actividad, actúa en este rol. En este caso se busca reducir las alternativas de decisión de la sociedad alineándose con una alternativa/decisión particular $y$, frecuentemente, asumiendo una posición política. Por último, el cuarto rol que plantea Pielke, Jr (2007) es el de "Promotor de Alternativas", en el cual se busca expandir el abanico de posibilidades de decisión disponibles. Quienes asumen este rol tienen una fuerte interacción con los actores de manera de desarrollar opciones que rompan "trampas de rigidez" y permitan alcanzar objetivos específicos.

Todos estos roles son importantes en cuanto a su capacidad de contribuir a un proceso de OTR. Por otra parte, como toda clasificación, fija límites arbitrarios en dimensiones continuas. El "árbitro científico" puede transformarse en un "abogado de una causa". ¿Cuándo se deja el rol de "científico puro" para pasar a ser árbitro? ¿Hasta dónde un "promotor de alternativas" expande opciones? 


\section{Desafíos}

Incorporar a la Ciencia en el proceso de OTR es el camino para abandonar el pensamiento mágico y construir alternativas basadas en las evidencias (empíricas y lógicas) y en la discusión de estas con el resto de los actores que participan. El aporte de la Ciencia y los científicos parece el camino más corto para minimizar los efectos negativos de los factores que generan deterioro en los sistemas socioecológicos y que, eventualmente, llevaron a muchas sociedades al colapso. Para ello, el SCyT debería ser capaz de establecer un dialogo con los actores en el territorio que haga posible reconocer los intereses y funciones del sistema socio-ecológico que están en discusión y proveer la información y guía necesarias para llevar adelante una negociación informada y transparente.

Incorporar al SCyT a los procesos de OTR enfrenta muchos desafíos y problemas. Una lista no exhaustiva incluye los conflictos de interés, el reduccionismo y la consecución de la inter / trans / multidisplinariedad. En gran medida, la importancia de la participación de los científicos está asociada a la credibilidad del SCyT. Obviamente, los científicos participarán en el proceso de OTR desde una posición ideológica. Cuanto más explícita sea la definición de esa posición, más clara será la contribución, siendo un primer paso valioso definir desde cuál de los cuatro roles que plantea Pielke Jr. (2007) se actúa. Los científicos, en tanto intelectuales, pueden asumir diferentes posiciones respecto de los actores. Gramsci (1972) separa a los intelectuales tradicionales (no asociados a un grupo, clase o actor social) de los orgánicos (vinculados a los intereses y valores de un grupo). Cuando este vínculo es explícito estamos frente a una situación virtuosa: la sociedad tiene claro en pos de qué valores o intereses aboga un científico. Cuando los intereses no son explícitos, los conflictos que se generan afectan seriamente la credibilidad. El conflicto de interés se asocia a la existencia de condicionantes (económicos o de otra índole) que podrían (se destaca el potencial) afectar la opinión de una persona. Un miembro del SCyT que tienen contratos (perfectamente legítimos) con un actor (e.g., una empresa cuyos intereses puede verse afectados por la localización o restricción de usos del suelo) no debería participar del proceso. En este caso se corre el riesgo de que quien dice actuar como "científico puro" o "árbitro" sea un "abogado de una causa particular". El SCyT debe estar muy atento a la identificación de estos conflictos en su seno y desarrollar mecanismos que los minimicen. Sin duda el co-gobierno universitario de algunas de las universidades latinoamericanas contribuye a la transparencia y a minimizar conflictos de interés.

El reduccionismo y la promoción de la multi / inter / transdisciplinariedad son aspectos que deben atenderse juntos. Abandonar el reduccionismo es una tarea enorme dado el tipo de formación disciplinaria desde la cual los académicos participan en el proceso de OTR (Levins 2015). Lograr una visión sistémica y dialéctica de la realidad que evite los "holismos místicos" requiere la integración de distintas visiones disciplinarias, las cuales muchas veces parten de marcos conceptuales distintos y no comparten un mismo lenguaje. La formación de profesionales capaces de interactuar con representantes de distintas disciplinas y de facilitar el proceso de integración es un punto a considerar seriamente. Programas a nivel de especialización de posgrado pueden jugar este papel. Sin embargo, la necesidad de establecer puentes que promuevan el diálogo entre disciplinas no debería ir desmedro de la promoción de una sólida base disciplinaria de los participantes en el proceso.

Agradecimientos. Agradezcolos comentarios y aportes de Esteban Jobbágy, Hernán Dieguez, Agustina García-Collazo y de un revisor anónimo. Este trabajo se llevó a cabo con la financiación del proyecto del Inter-American Institute for Global Change Research (IAI) CRN3095 el cual a su vez es financiado por la US National Science Foundation (Grant GEO-1128040) y con el aporte de proyectos FONCYT, UBACYT y PIP CONICET.

\section{REFERENCIAS}

Amdan, ML; R Aragón; EG JobbágY; JN Volante \& JM Paruelo. 2013. Onset of deep recharge and salt mobilization following forest clearing and cultivation in the Chaco plains (Argentina). Water Resources Research, 49:1-12.

CereiJido, M. 1994. Ciencia sin Seso Locura Doble. Siglo XXI Editores. México, D.F. Pp. 287.

Cereijido, M \& L Reinking. La ignorancia debida. Libros del Zorzal. Buenos Aires. Pp. 192

Diamond, J. 2006. Collapse: How Societies Choose to Fail or Survive. Penguin Books. England. Pp. 691. 
EzcurRA,E.1998. Delaschinampasalamegalópolis. Elmedioambienteenlacuenca deMéxico. FondodeCulturaEconómica. México, D.F. Pp. 130.

García-Collazo, MA; A Panizza \& JM Paruelo. 2013. Ordenamiento territorial de bosques nativos: Resultados de la zonificación realizada por provincias del norte Argentino. Ecología Austral, 23:97-107.

Gramsci, A. 1972. Los intelectuales y la organización de la cultura. Buenos Aires. Ed. Nueva Visión. Buenos Aires. Pp. 157.

GUNDERSON, LH \& CS HolLING. 2002. Panarchy: understanding transformations in human and natural systems. Island Press. Washington, D.C. Pp. 450.

LATOUR, B. 2012. Cogitamus: seis cartas sobre las humanidades científicas. Ed. Paidós. Buenos Aires. Pp. 200.

Levins, R. 2015. Una pierna adentro, una pierna afuera. CopIt ArXives \& Editora C3, México.

MAHner, M \& M Bunge. 1997. Foundations of Biophilosophy. Springer. Berlín. Pp. 423.

MacArthur, RH \& EO Wilson. 1967. The Theory of Island Biogeography. Princeton,NJ: Princeton UniversityPress.

Nosetto, MD; RA PÁez; SI BAllesteros \& EG JobbÁGy. 2015. Higher water-table levels and flooding risk under grain vs. livestock production systems in the subhumid plains of the Pampas, Agriculture, Ecosystems \& Environment, 206: 60-70.

Ostrom, E. 1999. Coping with tragedies of the Commons. Annual Review of Political Science, 2:493-535.

Paruelo, JM \& MR Aguiar. 2003. El impacto humano sobre los ecosistemas: el caso de la desertificación en Patagonia. Ciencia Hoy, 13:48-59.

Paruelo, JM. 2009. En relación con el artículo "El rol del ecólogo ante la actual crisis ambiental", de Gurvich et al. Ecología Austral, 19:255-258.

Paruelo, JM; EG Jobbágy; P Laterra; H Diéguez; MA García-Collazo; et Al. (eds.). 2015. Ordenamiento Territorial: Conceptos, Metodologías y Experiencias. Ed. FAO/ MAG/ UBA.

Pielke Jr., RA. 2007. The honest broker. Making sense of science in policy and politics. Cambridge University Press. Pp. 198.

Redman, CL. 1999. Human Impact on Ancient Environments. University of Arizona Press. Tucson. Pp. 239.

Rulli, MC; A SAVIORI \& P D'Odorico. 2013. Global land and water grabbing. Proceedings of the National Academy of Sciences, 110:892-896.

SANTONI, C; EG JobbáGy \& S CONTRERAs. 2010. Vadose zone transport in dry forests of central Argentina: Role of land use. Water Resources Research, 46:W10541. 